\title{
Phylogenetic investigation of endophytic Fusarium strain producing antimicrobial metabolite isolated from Himalayan Yew Bark
}

\author{
Tayung, K. ${ }^{1^{\star}}$, Barik, B. P. ${ }^{2}$, Jagadev, P. N. ${ }^{3}$ and Mohapatra, U. B. ${ }^{1}$ \\ ${ }^{1}$ Department of Botany, North Orissa University, Baripada-757003, Orissa, India. \\ ${ }^{2}$ Department of Bioinformatics, North Orissa University, Baripada-757003, Orissa, India. \\ ${ }^{3}$ Department of Bioinformatics, Orissa University of Agriculture and Technology, \\ Bhubaneswar- 751003, Orissa, India. \\ E-mail: kumanandbotnou@rediffmail.com
}

Received 14 February 2010; received in revised form 13 April 2010; accepted 22 April 2010

\begin{abstract}
An endophytic fungus, Fusarium sp. was isolated from yew bark of eastern Himalaya. Ethyl acetate extract from its fermentation broth displayed considerable antimicrobial activity against three Gram-positive bacteria (Staphylococcus aureus, Bacillus subtilis and Staphylococcus epidermidis), three Gram-negative bacteria (Klebsiella pneumoniae, Escherichia coli and Shigella flexneri) and two pathogenic fungi (Candida albicans and Candida tropicalis). The metabolite showed highest inhibition zone against $K$. pneumoniae $(27 \mathrm{~mm})$ and lowest against $C$. albicans (10 mm). Based on BLAST search analysis of ITS rDNA sequence, the fungus was identified as Fusarium solani (Mart.) Sacc. Phylogenetic trees were generated by four different methods. Phylogenetic tree generated by UPGMA method was used to establish possible phylogenetic relationships of the fungus with other $F$. solani isolates those exist as endophytes, pathogens and saprotrophs taken from database. The generated tree showed that all $F$. solani strains have a common endophytic ancestry which gave rise to six clades that radiate into four evolutionary lineages. The possible phylogenetic relationships of $F$. solani that exist in different lifestyle have been discussed in each clade.
\end{abstract}

Keywords: antimicrobial activity, endophytic fungus, F. solani, ITS rDNA sequence, phylogenetic tree

\section{INTRODUCTION}

Endophytic fungi are microbes that colonized healthy inner plant tissues without causing any disease symptoms (Wilson, 1995). Studies on endophytic fungal diversity in plant kingdom have indicated their presence in several trees and shrubs (Rodrigues, 1996; Raviraja, 2005). Interest in fungal endophytes associated with medicinal plants has increased in the last decade, due to isolation of new and interesting bioactive metabolites of multiple applications (Tan and Zou, 2001; Strobel and Daisy, 2003). Several bioactive metabolites including some compounds with antibacterial and antifungal activities have been reported from endophytic fungi associated with medicinal plants (Li et al., 2005; Xu et al., 2008; Gong and Guo, 2009). In our screening program for antimicrobial metabolites from endophytic fungi associated with medicinal plants, an endophyte identified as Fusarium sp. was isolated from bark of Himalayan yew, which is high altitude gymnosperm plant with anticancer property. The crude extract of this fungus exhibited potent antimicrobial activity against some clinically significant microorganisms. Several Fusarium species isolated as endophytes have been reported to produce metabolites with antimicrobial and anticancer activity (Xu et al., 2008; Deng et al., 2009; Gong and Guo, 2009). However, their occurrence as endophytes has been speculated by many researchers because of their association in causing diseases to many plants and animals. Moreover, there is speculation among the researchers about their nature of association in plants, as most Fusarium species are commonly encountered in soil as saprophytes, pathogens and are also associated with serious invasive mycoses in immunocompromised patients (Summerbell, 2003). Such occurrence of Fusarium in different forms is often confusing, as they resemble similar morphological features. Species identification by morphological traits is problematic because mycelial pigmentation, shape and size of conidia are unstable and highly dependent on composition of media and environmental conditions. So, methods are needed to distinguish between closely related species that occur in different habitat. Molecular techniques for fungal identification and to investigate genetic variability within species are increasingly used during the last decade. Of particular importance is the internal transcribed spacer (ITS) which is located between the nuclear genes for ribosomal RNA. The rDNA internal transcribed spacer (ITS) region sequences are now largely used for identification and phylogenetic analysis of fungi (Guo et al., 2001).

Therefore, the objectives of the present study was to investigate the possible phylogenetic relationship of an 
endophytic Fusarium strain with other isolates exist as endophytes, saprophytes and pathogens and to determine the antimicrobial potential of the crude metabolite produced by the fungus against some clinically significant microorganisms.

\section{MATERIALS AND METHODS}

\section{Sample collection and isolation of endophytic fungi}

Bark of Taxus baccata commonly known as Himalayan yew was collected from Dibang valley of eastern Himalaya, a part of Indo-Burma biodiversity hotspot, located between latitude $28^{\circ} 20^{\prime} 7^{\prime \prime} \mathrm{N}$ and longitude $95^{\circ}$ 46'38" E. To obtain fungal endophytes, bark were sterilized by immersing them sequentially in $70 \%$ ethanol for $3 \mathrm{~min}$ and $0.5 \% \mathrm{NaOCl}$ for $1 \mathrm{~min}$ and rinsed thoroughly with sterile distilled water. The excess water was dried under laminar airflow chamber. Then, with a sterile scalpel, outer tissues were removed and the inner tissues were carefully dissected and placed on petri-plates containing potato dextrose agar (PDA) and water agar (WA) medium. The plates were sealed with parafilm and incubated in BOD incubator at $24 \pm 1{ }^{\circ} \mathrm{C}$ for days unti fungal growth appeared on the plates. Hypal tips of the fungi growing out of the plated bark were transferred with a fine sterile needle to freshly prepared potato dextrose agar slants. The endophytes were checked for purity and pure cultures were stored at $4{ }^{\circ} \mathrm{C}$ for further analysis. The efficiency of surface sterilization techniques was tested as described by Schultz et al. (1998).

\section{Metabolite extraction and antimicrobial study}

The endophytes were evaluated for their antimicrobial potential against some clinically significant human pathogens. Among the endophytes, a strain exhibiting good antimicrobial activity was selected and cultivated on potato dextrose broth by placing agar blocks of actively growing culture in $500 \mathrm{~mL}$ Erlenmeyer flasks containing $200 \mathrm{~mL}$ of the medium. The flasks were incubated in BOD shaking incubator for 3 weeks at $24 \pm 2{ }^{\circ} \mathrm{C}$ with periodic shaking at $150 \mathrm{rpm}$. The fermentation broth was then extracted thrice with ethyl acetate at room temperature. The organic solvent was evaporated in vacuum evaporator and the resultant compound was dried with $\mathrm{MgSO}_{4}$ and concentrated to yield the crude metabolites. The metabolites were dissolved in Dimethyl sulphoxide (DMSO) for antimicrobial bioassay. Eight human pathogenic microorganisms that include three Grampositive bacteria (Staphylococcus aureus, Bacillus subtilis and Staphylococcus epidermidis), three Gram-negative bacteria (Klebsiella pneumoniae, Escherichia coli and Shigella flexneri) and two pathogenic fungi (Candida albicans and Candida tropicalis) were used as test pathogens. The inhibitory effect of the crude extract was tested by agar cup diffusion assay at a concentration of 1 $\mathrm{mg} / \mathrm{mL}$. The magnitude of antimicrobial actions was assessed by the diameter $(\mathrm{mm})$ of inhibition zones relative to those of positive and negative controls. Tetracycline and Fluconazole were co-assayed as positive antimicrobial references with DMSO as negative control.

\section{Isolation of genomic DNA, PCR amplification and sequencing}

Total genomic DNA was extracted from mycelia of the fungus grown on potato dextrose agar medium by using CTAB method (Cai et al., 2006). DNA amplification was performed by PCR. The PCR was set up using the following components: $5 \mu \mathrm{L}$ Buffer (10X), $3 \mu \mathrm{L} \mathrm{MgCl}_{2}(25$ $\mathrm{mM}), 1 \mu \mathrm{L}$ dNTPs (10 mM), $1.5 \mu \mathrm{L}$ Taq Polymerase (5 U), $1.5 \mu \mathrm{L}$ Forward Primer $(10 \mu \mathrm{M}), 1.5 \mu \mathrm{L}$ Reverse Primer $(10 \mu \mathrm{M}), 3 \mu \mathrm{L}$ DNA template and $34.7 \mu \mathrm{L}$ distilled water. The PCR condition was run in such a way, where initial denaturation was at $95{ }^{\circ} \mathrm{C}$ for $5 \mathrm{~min}$. Denaturation, annealing and elongation were done at $95^{\circ} \mathrm{C}$ for $1 \mathrm{~min}, 52$ ${ }^{\circ} \mathrm{C}$ for $30 \mathrm{sec}$ and $72{ }^{\circ} \mathrm{C}$ for $1 \mathrm{~min}$ respectively in 45 cycles. Final extension was done at $72{ }^{\circ} \mathrm{C}$ for $10 \mathrm{~min}$ and hold at $4{ }^{\circ} \mathrm{C}$ forever. For amplification of ITS-rDNA region ITS4 and ITS5 primers were used according to the method described by White et al. (1990). The PCR product, spanning approx. 500-600 bp was checked on $1 \%$ agarose electrophoresis gels. It was then purified using quick spin column and buffers (washing buffer and elution buffer) according to the manufacturer's protocol (QIA quick gel extraction kit Cat No. 28706). DNA sequencing was performed using the above mentioned primers in an Applied Biosystem 3130xl analyzer.

\section{Phylogenetic study}

Phylogenetic analysis was conducted based on ITS sequences of the fungus (partial 18S, ITS1, 5.8S complete, ITS2 and 28S partial genes) and similar sequences of $F$. solani isolates retrieved from Genbank database randomly. During the retrieval process hosts, geographical locations and nature of occurrence of the isolates were considered. The sequence similarity searches were performed by BLAST algorithm and aligned using clustalw. Phylogenetic trees were generated by four different methods (UPGMA, NJ, MP and Minimum Evolution) by using MEGA4.0 (Tamura et al., 2007). Tree showing consistency in topology was considered for present study.

\section{RESULTS AND DISCUSSION}

In the present investigation we obtained an endophytic fungus producing antimicrobial metabolite harboring in the bark of Himalayan yew (Taxus baccata). In addition to morphological characterization, molecular analysis was carried out to confirm the identification of the fungus. Based on BLAST search analysis of ITS rDNA sequence, the fungus was identified as Fusarium solani (Mart.) Sacc. A living culture of the isolate is deposited in Microbial Type Culture Collection (MTCC) and Gene bank, Institute of Microbial Technology (IMTECH) Chandigarh, India with accession no. 9622. The sequence has also been deposited in Genbank of NCBI with accession no. 
FJ719812. Fusarium solani is a fungus that is widely distributed in nature and is able to cause many plant diseases with important economic impacts and also severe human infections (Guarro and Gene, 1995). However, at present they have been isolated as endophytes from many plant species. Occurrence of $F$. solani as endophyte has also been reported from other yew species with anticancer activity (Chakravarthi et al., 2008; Deng et al., 2009). This suggests possible allopatric speciation of this fungus based on different geographical locations and ecological niches. The crude metabolite extracted from fermentation broth of the fungus exhibited strong to moderate antimicrobial activity against all the test pathogens (Table 1). To assess the magnitude of antimicrobial action, the metabolites was co-assayed with two reference antibiotics i.e. Tetracycline as antibacterial and Fluconazole as antifungal agent. The metabolite showed highest zone of inhibition against $K$. pneumoniae $(27 \mathrm{~mm})$ followed by $S$. flexneri $(24 \mathrm{~mm})$ whose antibacterial activity was almost similar to that of the positive control, Tetracycline (Fig. 1). Among the bacterial pathogens, the metabolite showed lowest activity against E. coli $(16 \mathrm{~mm})$ but the activity was greater than that of the co-assayed antibiotics. Further, the metabolite exhibited strong antifungal activity against $C$. albicans $(20 \mathrm{~mm})$ but low activity against $C$. tropicalis $(10 \mathrm{~mm})$. Both these pathogens were resistant to the reference antifungal agent, Fluconazole (Fig. 2). Similarly, metabolites derived from other endophytic Fusarium species associated with medicinal plants have also displayed strong antimicrobial and antibacterial activity (Wang et al., 2007; Xu et al., 2008). Such results support the assumption that endophytic fungi are repository of novel secondary metabolites for potential therapeutic uses (Tan and Zou, 2001).

Phylogenetic trees were generated by using four different methods (UPGMA, NJ, MP and Minimum Evolution) based on ITS rDNA sequences of $F$. solani obtained from various environmental sources and locations illustrating their possible phylogenetic relationships at intraspecific level (Fig. 3). The tree generated by UPGMA method was considered because of its consistency in topology. In the present study three different groups of $F$. solani constituting endophytes, saprophytes and pathogens to plants, animals and human were considered (Table 2). The tree showed that all $F$. solani strains have a common endophytic ancestry. This ancestral endophytic $F$. solani has given rise to six clades that radiate into four evolutionary lineages. Clade I consisted of five $F$. solani isolates comprising four endophytic and one pathogenic species. This clade indicates that pathogenic strain has evolved to endophytic species retaining its ancestral gene genealogy. The transformation of pathogenic to endophytic character may be attributed to the loss of virulence due to mutation. Since such mutational frequency is very common in genus Fusarium under different cultural and environmental condition (Miller, 1945). Clade II contained three isolates consisting of two pathogenic and one saprophytic species. The clade showed parallel evolution of pathogenic and endophytic strains from a pathogenic species. This suggests transitional evolution of pathogenic species to saprophytic habit before it has transformed towards endophyte. Clade III displayed co-existence of endophytic and saprophytic $F$. solani species. Such coevolution may be due to the fact that several endophytes and saprotrophs have intimate link which have been postulated by various workers based on their morphological evidences. Further, ribosomal DNA-based sequence comparison and phylogenetic relationships from 99 fungal isolates (endophytes, mycelia sterilia, and saprotrophs) recovered from leaves and twigs of Magnolia liliifera suggest that these fungal taxa possibly exist as endophytes and saprotrophs (Promputtha et al., 2006). Such coevolution has also been manifested in clade IV.

Table 1: Antimicrobial activity of the crude metabolite extracted from endophytic Fusarium

\begin{tabular}{lccc}
\hline Test pathogen & $\begin{array}{c}\text { Zone of inhibition }(\mathrm{mm}) \\
(1 \mathrm{mg} / \mathrm{mL})\end{array}$ & $\begin{array}{c}\text { Tetracycline } \\
(30 \mathrm{mcg} / \mathrm{disc})\end{array}$ & $\begin{array}{c}\text { Fluconazole } \\
(10 \mathrm{mcg} / \mathrm{disc})\end{array}$ \\
\hline Staphylococcus aureus & $18.6 \pm 1.1$ & $22.0 \pm 0$ & - \\
Bacillus subtilis & $18.3 \pm 1.5$ & $24.3 \pm 0.5$ & - \\
Staphylococcus epidermidis & $20.3 \pm 0.5$ & $24.0 \pm 0.5$ & - \\
Klebsiella pneumonia & $27.0 \pm 1.7$ & $30.0 \pm 0$ & - \\
Escherichia coli & $16.3 \pm 0.5$ & $08.6 \pm 1.1$ & - \\
Shigella flexneri & $24.3 \pm 1.1$ & $25.0 \pm 0$ & - \\
Candida albicans & $20.3 \pm 0.5$ & - & - \\
Candida tropicalis & $10.6 \pm 1.1$ & - & - \\
\hline
\end{tabular}

${ }^{ \pm}$SD- standard deviation; ${ }^{-}$No inhibition.

Negative control: Dimethly sulphoxide (the medium to dissolve the crude metabolites) $100 \mu \mathrm{L} /$ well.

Co-assayed antibiotics: Tetracyline $(30 \mathrm{mcg} / \mathrm{disc})$ for bacteria; Fluconazole $(10 \mathrm{mcg} / \mathrm{disc})$ for fungi 
Parallel evolution of saprophytic and pathogenic strains was seen in clade $V$. This also suggests possible coexistence between these species. Such phenomenon is well observed in genus Fusarium where saprophytic species readily colonizes diseased root and stem materia making it difficult to isolate the pathogen that caused the disease. Clade VI showed that the highly evolved pathogenic strains have descended from an endophytic species conserving its remote ancestral endophytic habit. This endophytic strain (Accession no FJ719812) was isolated from yew bark of eastern Himalaya. The ancestral conservative nature of its strain may be attributed to geographical isolation barrier of this region.

The present investigation is a first report of occurrence endophytic $F$. solani in yew bark of eastern Himalaya producing antimicrobial metabolite and an attempt to study its possible evolutionary relationship considering isolates exist as endophytes saprophytes and pathogens. Currently we are working to characterize the active metabolite produced by the endophytic fungus.

Table 2: List of F.solani strains and Genbank accession numbers of ITS sequences used in this study

\begin{tabular}{lllll}
\hline Species & Source & Habitat & Country & Genbank Accession \\
\hline F. solani & Taxus baccata & Endophytic & India & FJ719812* \\
F. solani & Taxus chinesis & Endophytic & China & EU442277.1 \\
F. solani & Amona Squamosa & Endophytic & China & EF488412 \\
F. solani & Amona Squamosa & Endophytic & China & EF488413 \\
F. solani & Campotheca acuminate & Endophytic & China & EF062312 \\
F. solani & Campotheca acuminate & Endophytic & China & FM179605.1 \\
F. solani & Nothapodytes nimmoniana & Endophytic & India & FJ158121.1 \\
F. solani & Nothapodytes nimmoniana & Endophytic & India & FJ158119 \\
F. solani & Cucurbit fruit & Phytopathogenic & New Zealand & DQ094647 \\
F. solani & Ailanthus sp. & Phytopathogenic & USA & DQ094649 \\
F. solani & Cassava root & Phytopathogenic & Benin & DQ094650 \\
F. solani & Citrus tree & Phytopathogenic & Colombia & DQ094655 \\
F. solani & Cancer patient & Human Pathogenic & USA & DQ094656 \\
F. solani & Human cornea & Human Pathogenic & Germany & DQ094422.1 \\
F. solani & Tree Fish eye & Animal Pathogenic & USA & DQ094414 \\
F. solani & Shrimp & Animal Pathogenic & Hawaii & DQ094330 \\
F. solani & Soil & Saprophytic & Thailand & DQ094722 \\
F. solani & Soil & Saprophytic & South Africa & DQ094700 \\
F. solani & Soil & Saprophytic & New Caledonia & DQ094713 \\
F. solani & Soil & Saprophytic & Bulgaria & AB508989.1 \\
F. solani & Soil & Saprophytic & Japan & FJ460583.1 \\
F. solani & Soil & Saprophytic & GK & EF621488.1 \\
F. solani & Compost & Saprophytic & Spain & AM412637 \\
F. solani & Aquarium Land & Saprophytic & &
\end{tabular}

${ }^{*}$ Accession no. of the fungus used in the present study

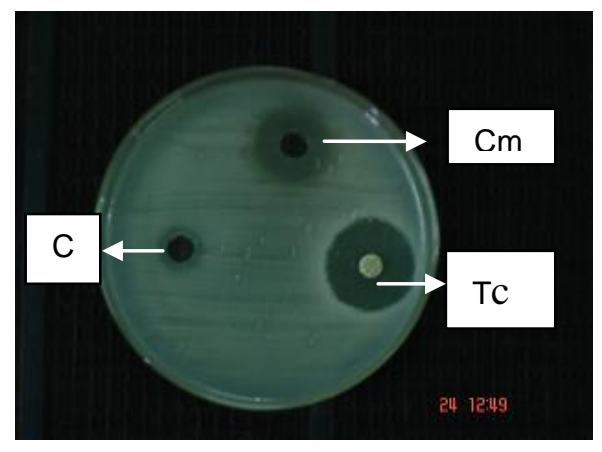

Figure 1: Antibacterial activity of the crude metabolites (Cm) against Staphylococcus epidermidis. Coassayed antibiotic Tetracycline (Tc) and control (C)

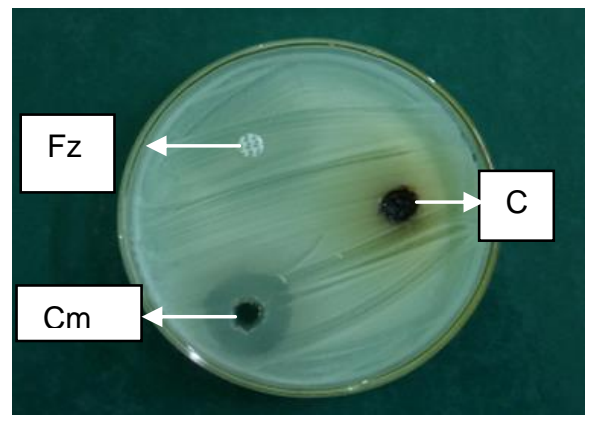

Figure 2: Antifungal activity of the crude metabolites $(\mathrm{Cm})$ against Candida albicans. Co- assayed antibiotic Fluconazole (Fz) and control (C) 


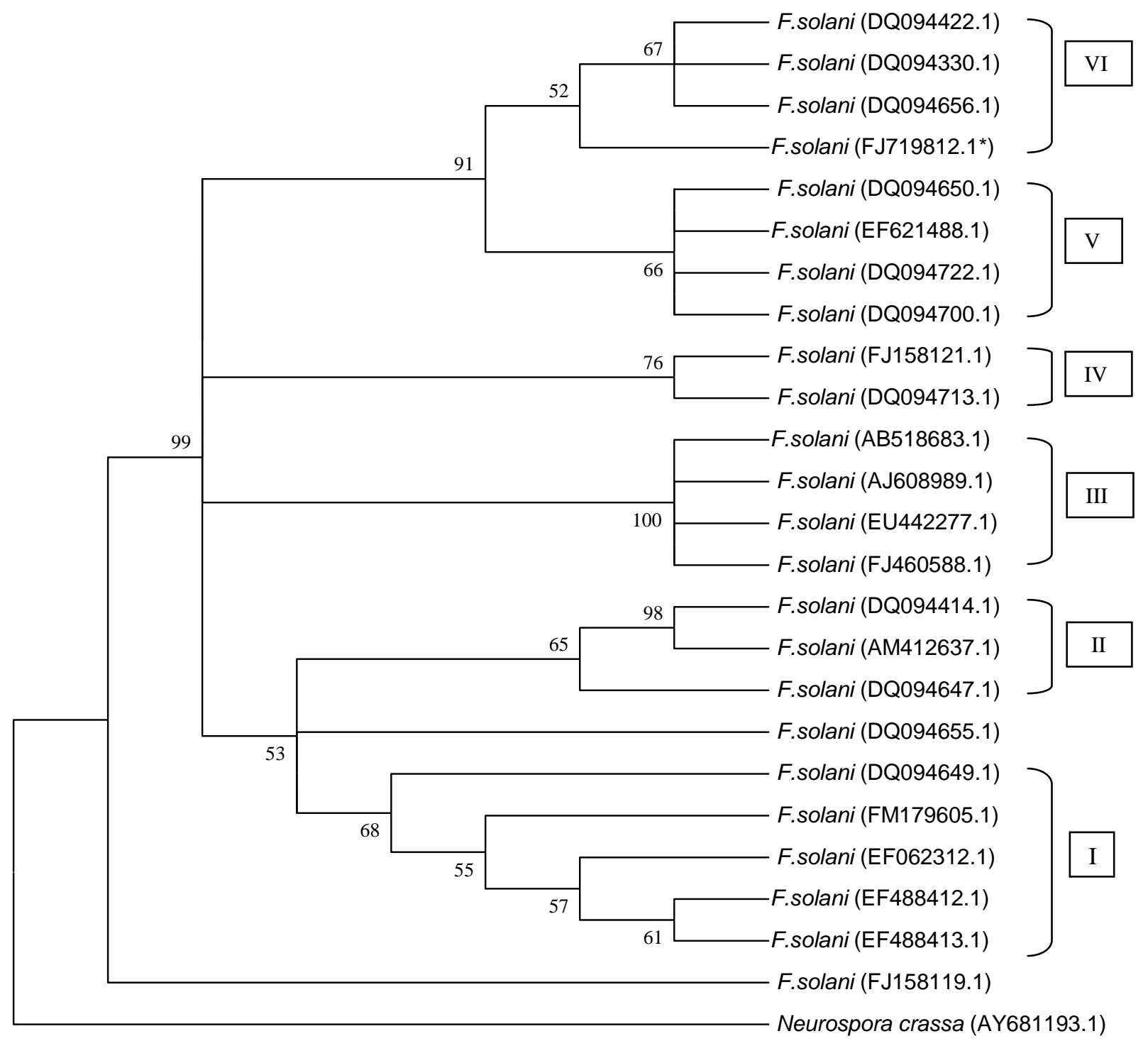

*Accession number of the endophytic fungus in the present study.

Figure 3: Phylogenetic tree showing evolutionary relationships of $24 \mathrm{~F}$. solani strains that exist in different forms. The evolutionary history was inferred using the UPGMA method. The optimal tree with the sum of branch length = 0.41451003 is shown. The percentage of replicate trees in which the associated taxa clustered together in the bootstrap test (500 replicates) is shown next to the branches. The accession numbers of the strains are given in parenthesis. Neurospora crassa is taken as outgroup

\section{ACKNOWLEDGEMENT}

The authors are grateful to Dr. Shenoy B. D. (Scientist), Institute of Microbial Technology, Chandigarh, India for his help and co-operation in DNA sequencing of the fungus.

\section{REFERENCES}

Cai, L., Jeewon, R. and Hyde, K. D. (2006). Phylogenetic investigation of sordariaceae based on multiple gene sequences and morphology. Mycological Research 110, 137-150.

Chakravarthi, B. V. S. K., Das, P., Surendranath, K., Karande, A. A. and Jayabaskaran, C. (2008). 
Production of paclitaxel by Fusarium solani isolated from Taxus celebica. Journal of Bioscience 32, 1-9.

Deng, B. V., Liu, K. H., Chen, W. Q., Ding, X. W. and Xie, X. C. (2009). Fusarium solani, Tax-3, a new endophytic taxol-producing fungus from Taxus chinensis. World Journal of Microbiology and Biotechnology 25, 139-143.

Gong, L. J. and Guo, S. X. (2009). Endophytic fungi from Dracaena cambodiana and Aquilaria sinensis and their antimicrobial activity. African Journal of Biotechnology 8, 731-736.

Guarro, J. and Gene, J. (1995). Opportunistic Fusarial infections in humans. European Journal of Clinical Microbiology and Infectious Diseases 14, 741-754.

Guo, L.D., Hyde, K.D. and Liew, E.C.Y. (2001). Detection and identification of endophytic fungi within frond tissues of Livistona chinensis based on rDNA sequence. Molecular Phylogenetics and Evolution 20, 1-13.

Li, Y., Song, Y. C., Liu, J. Y., Ma, Y. M. and Tan, R. X. (2005). Anti-Helicobacter pylori substances from endophytic fungal cultures. World Journal of Microbiology and Biotechnology 21, 553-558.

Miller, J. J. (1945). Studies on the Fusarium of muskmelon wilt: pathogenic and cultural studies with particular reference to the cause and nature of variation in the causal organism. Canadian Journal of Research 23, 16-43.

Promputtha, I., Lumyong, S., Dhanasekaran, V., McKenzie, E. H. C., Hyde, K. D. and Jeewon, R. (2006). A phylogenetic evaluation of whether endophytes become saprotrophs at host senescence. Microbial Ecology 53, 579-590.

Raviraja, N. S. (2005). Fungal endophytes in five medicinal plant species from Kudremukh Range, Western Ghats of India. Journal of Basic Microbiology 45, 230-235.

Rodrigues, K. F. (1996). Fungal endophytes of palms. In: Endophytic fungi in grasses and woody plants. Redlin, S. C. and Carris, L. M. (eds.). APS Press, California. pp. 121-132.

Schultz, B., Guske, S., Dammam, U. and Boyle, C. (1998). Endophyte-host interactions II. Defining symbiosis of the endophyte-host interaction. Symbiosis 25, 213-227.

Strobel, G.A. and Daisy, B. (2003). Bioprospecting for microbial endophytes and their natural products. Microbiology and Molecular Biology Reviews 67, 491502.

Summerbell, R. C. (2003). Aspergillus, Fusarium, Sporothrix, Piedraia, and their relatives, In: Pathogenic fungi in humans and animals. Howard, D. H. (eds.). Marcel Dekker, New York, pp. 237-498.

Tamura, K., Dudley, J., Nei, M. and Kumar, S. (2007). MEGA4: Molecular Evolutionary Genetics Analysis (MEGA) software version 4.0. Molecular Biology and Evolution 24, 1596-1599.

Tan, R. X. and Zou, W. X. (2001). Endophytes: A rich source of functional metabolites. Natural Product Report 18, 448-459.
Wang, F. W., Jiao, R. H., Cheng, A. B., Tan, S. H. and Song, Y. C. (2007). Antimicrobial potentials of endophytic fungi residing in Quercus variabilis and brefeldin A obtained from Cladosporium sp. World Journal of Microbiology and Biotechnology 23, 79-83.

White, T. J., Bruns, T. D., Lee, S. and Taylor, J. W. (1990). Amplification and direct sequencing of fungal rRNA genes for phylogenetics. In: Protocols: A guide to methods and application. Innis, M. A., Gelfand, D. H., Sninsky, J. and White, T. J. (eds.). PCR. Academic Press, San Diego, pp. 315-322.

Wilson, D. (1995). Endophyte-the evolution of a term, and clarification of its use and definition. Oikos 73, 274276.

Xu, L., Zhou, L., Zhao, J., Li, J., Li, X. and Wang, J. (2008). Fungal endophytes from Dioscorea zingiberensis rhizomes and their antibacterial activity. Letters in Applied Microbiology 46, 68-72. 